\title{
Simple determination of photosynthetic efficiency and photoinhibition of Dunaliella tertiolecta by saturating pulse fluorescence measurements
}

\author{
J. W. Hofstraat ${ }^{1, *}$, J. C. H. Peeters ${ }^{1}$, J. F. H. Snel ${ }^{2, * *}$, C. Geel ${ }^{2}$ \\ 'Tidal Waters Division, Public Works Department, PO Box 20907, 2500 EX The Hague, The Netherlands \\ ${ }^{2}$ Department of Plant Physiology, Wageningen Agricultural University, Arboretumlaan 4, 6703 BD Wageningen, \\ The Netherlands
}

\begin{abstract}
Saturating pulse fluorescence measurements, well known from studies of higher plants, were applied to cultures of the chlorophyte Dunaliella tertiolecta. Photochemical and non-photochemical quenching, and the photochemical yield and photochemical efficiency of photosystem II, were determined for cultures of $D$. tertiolecta growing under varying light intensities. Photosynthetic efficiencies appeared to correlate well with growth rates determined for the $D$. tertiolecta cultures. The photochemical yield provides a measure of photoinhibition effects. The results indicate that the saturating pulse fluorescence method may be successfully used to determine photosynthetic characteristics of phytoplankton. The technique is simple to apply and can be adapted for photosynthetic measurements in situ, i.e. on phytoplankton in their natural habitat.
\end{abstract}

KEY WORDS: Dunaliella tertiolecta Fluorescence P Photoinhibition Photosynthesis - Photosynthetic efficiency $\cdot$ Phytoplankton - Saturating pulse fluorescence

\section{INTRODUCTION}

Fluorescence measurements have become widely applied in the determination of phytoplankton biomass. Based on the registration of fluorescence emission and excitation characteristics of phytoplankton communities, taxa can be roughly identified (Yentsch \& Yentsch 1979, Yentsch \& Phinney 1985a, b, Hilton et al. 1989). The measurement of chlorophyll fluorescence intensity has found general acceptance for the estimation of autotrophic phytoplankton biomass (Heaney 1978, Butterwick et al. 1982, Falkowski \& Kiefer 1985). An important advantage of this approach

\footnotetext{
- Present address: Akzo Research Laboratories Arnhem, Department CRL, PO Box 9300, 6800 SB Arnhem, The Netherlands

- Addressee for correspondence
}

is that in situ measurements on photosynthetic organisms are possible. To gain insight into the distribution (both horizontally, over large areas, and vertically, in the water column) of phytoplankton, in situ measurements are indispensable.

It appears, however, that the correlation between chlorophyll a fluorescence intensity and phytoplankton biomass, usually approximated by chemical measurement of the total amount of extracted chlorophyll $a$, is, in many instances, not very good (Falkowski \& Kiefer 1985). In particular, when one tries to correlate data acquired under different circumstances, e.g. at different depths, in different water masses or at different times of the day, the quantitative agreement between the chlorophyll a concentrations as determined by extraction and by fluorescence may be poor. This is mainly due to the fact that the fluorescence which is measured predominantly stems from chloro- 
phyll a molecules that are part of the photosynthetic apparatus of the cell; this apparatus is a dynamic system which can respond rapidly to changes in environmental circumstances. Pollutant stress, light intensity and temperature may thus strongly influence the fluorescence yield (Falkowski \& Kiefer 1985, Falkowski et al. 1986, Lichtenthaler \& Rinderle 1988). Thus, chlorophyll a fluorescence intensity, which can be examined by determination of the amount of fluorescence per molecule of chlorophyll a (i.e. the fluorescence quantum yield), can also be used to obtain information on the condition of the photosynthetic apparatus. This means that, in addition to estimates of total biomass, other information can be derived from phytoplankton fluorescence characteristics: estimates can be obtained of photosynthetic efficiency and, via that, of primary production and environmental stress. These aspects are considered in more detail in the following section.

The present paper describes the principles and applications of the saturating pulse fluorescence technique for determination of photosynthetic characteristics of phytoplankton. This method has mainly been applied to investigate higher plants, and can be used to obtain a quantitative estimate of the photochemical efficiency of the photosynthetic system (Schreiber 1983, Renger \& Schreiber 1986). Photoinhibitory effects and the amounts of photochemical and nonphotochemical quenching can also be determined (Schreiber et al. 1986). Measurement of the photochemical efficiency can be done directly and is suitable for in situ application (Genty et al. 1989, Falkowski \& Kolber 1990). As such, it has important advantages over ${ }^{14} \mathrm{C}$-incorporation measurement, which is largely agreed upon as the standard method for determining photochemical efficiency when the phytoplankton concentration is low, as is generally the case in marine applications (Peterson 1980, Dring \& Jewson 1982). For higher plants and in eutrophied situations, oxygen measurements can also be used which are much more easily applicable from an experimental point of view (Bryan et al. 1976). Determination of photoinhibitory effects and of photochemical and non-photochemical quenching requires dark adaptation prior to measurement, and is hence less suited for dynamic in situ measurements.

Experiments in this sudy were done on Dunaliella tertiolecta laboratory cultures with a dedicated, commercially available instrument that was designed for measurements on leaves (Schreiber 1986). The applicability of the saturating pulse fluorescence technique for marine phytoplankton will be demonstrated, and modifications that are required to make the instrument suitable for in situ purposes will be discussed. Firstly, however, the principles of saturating pulse fluorescence measurement are described.

\section{MEASUREMENT OF SATURATING PULSE FLUORESCENCE}

The fluorescence that is measured at ambient temperatures stems almost exclusively from chlorophyll associated with the antennae of photosystem (PS) II. The fluorescent yield of PS I is low because photochemistry here is a particularly efficient competitor, in addition to the other non-radiative decay processes such as thermal emission and triplet formation, unless measurements are done at low temperatures (Strasser \& Butler 1977, Briantais et al. 1986). Chlorophyll associated with the photochemical reaction centres represents only a very small fraction of the total chlorophyll content of phytoplankton and, in addition, has a very low fluorescence quantum yield.

The fluorescence yield of PS II chlorophyll, however, is highly variable. It is strongly influenced by the physiological state of the phytoplankton. The excited states that are produced by light absorption can be deactivated by 4 processes: (1) photosynthetic energy conversion, which requires energy transfer to the reaction center and subsequent electron transport followed by interaction with PS I, (2) triplet formation, (3) radiationless processes and (4) radiative transfer, the process that is accompanied by fluorescence. The fluorescence emission hence provides information on the photochemical processes in PS II. When the photochemical reaction centers are 'open', i.e. available to efficiently trap the excited state energy and to be involved in photochemical energy conversion, the yield of the nonphotochemical processes ( 2 to 4 ) will be low. On the other hand, when the reaction centers are closed the photochemical yield is very low and the non-photochemical processes become important. The fluorescence yield therefore varies inversely with the yield of photochemistry: it consists of a constant part and a variable part, which is determined by the state of the photochemical reaction center (open or closed). The state of the reaction centers is influenced by environmental circumstances (light history, nutrient status, presence of pollutant stress). It is just this dependence which makes simple, straightforward, in vivo fluorescence intensity measurements difficult to use for phytoplankton chlorophyll determinations.

On the other hand, fluorescence measurements can be used to monitor photosynthetic processes. The saturating pulse method that is used in this paper allows information on these processes to be obtained by application of a refined experimental scheme. In Fig. 1 the principles of the saturating pulse method are illustrated. The method is based on the application of 3 different excitation schemes to the phytoplankton. The chlorophyil fluorescence is measured using a lowpower, modulated, light-emitting diode for excitation. 
Fig. 1. Schematic representation of the saturating pulse fluorescence method (Van Kooten \& Snel 1990). Five different states of the photosynthetic system are defined in the course of a full experiment. At the onset of the experiment the dark-adapted states $F_{0}(1)$ and $F_{\mathrm{m}}(2)$ are defined by using modulated measuring light (ML) only and an additional saturating pulse (SP). respectively, for the 2 parameters. Next, the sample is illuminated with actinic light ( $\mathrm{AL}$ ) and a fluorescence induction (or Kautsky) curve is obtained. In the steady state $F(3)$ and $F_{m}^{\prime}(4)$ can be determined by again measuring with modulated measuring light and an additional saturating pulse, now in combination with the actinic light. Finally, the actinic light source is extinguished and far-red illumination (FR) is applied to reach $F_{0}{ }^{\prime}(5)$. To determine the photochemical efficiency according to the method of Genty et al. (1989), it is sufficient to determine $F_{\mathrm{m}}$ and $F$ under steady-state conditions

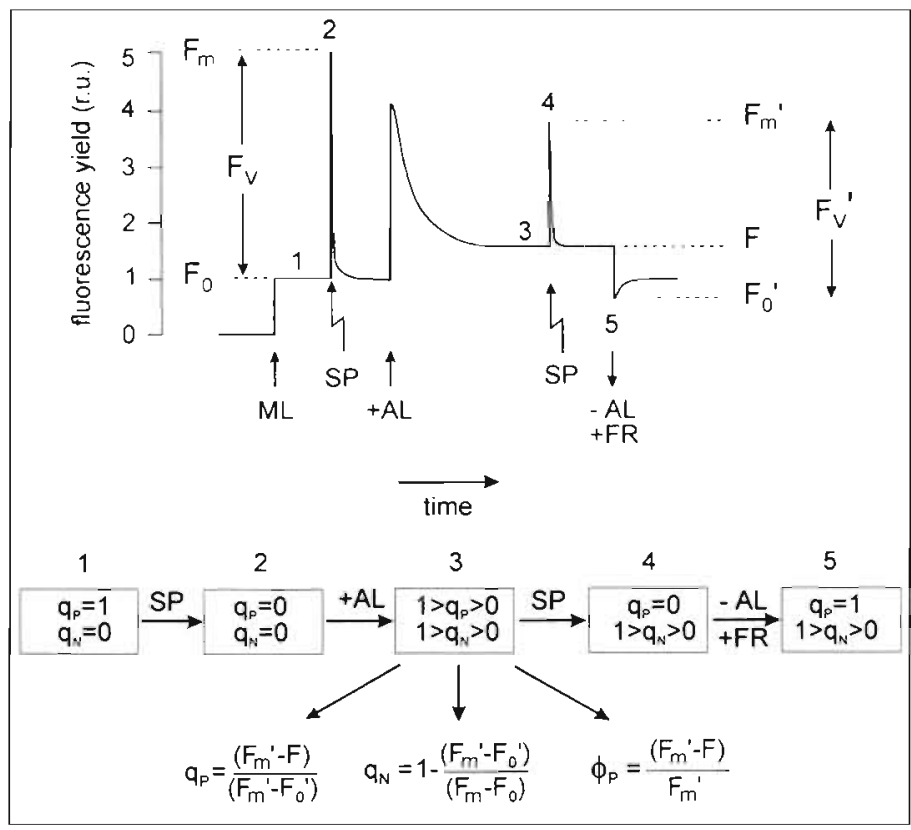

The fluorescence induced by this probe beam is selectively registered by application of phase resolved detection. In addition, a constant, variable-intensity, white light source may be used for actinic illumination of the sample. To investigate the photosynthetic system, a very high intensity pulse of white light may also be applied, which can saturate the photochemical reaction centers.

The full technique shown in Fig. 1 provides 4 useful parameters (see also Genty et al. 1989):

(1) Photochemical quenching, $q_{\mathrm{p}}$. Photochemical quenching of fluorescence is caused by the transfer of excited state energy to the photochemical reaction centers, where it is available for photochemical energy conversion. In dark-adapted phytoplankton the PS II reaction centers are all open and photochemical quenching is maximal. The fluorescence intensity at this point is designated $F_{0}$. When a short pulse of intense light is given, all PS II reaction centers are closed within a few hundred ms. During the pulse the fluorescence intensity reaches its maximum value, $F_{\mathrm{m}}$. During illumination with the actinic light source, or under ambient light, $q_{\mathrm{p}}$ can be determined by measuring the normalised difference between the maximum and the actual fluorescence yield:

$$
q_{\mathrm{p}}=\frac{F_{\mathrm{m}}{ }^{\prime}-F}{F_{\mathrm{rl}}{ }^{\prime}-F_{0}^{\prime}}
$$

where $F_{\mathrm{m}}{ }^{\prime}=$ the maximum fluorescence intensity that is obtained by saturation of the reaction centers under steady-state conditions; $F=$ the normal fluorescence intensity in the steady state; and $F_{0}{ }^{\prime}=$ the fluorescence intensity that is obtained directly after the actinic light source has been switched off; in addition, illumination by far-red light is applied, which is predominantly absorbed by PS I and results in reopening of intact PS II reaction centers by reoxidation of the quinone acceptor $Q_{A}$.

(2) Non-photochemical quenching, $q_{\mathrm{N}}$. Non-photochemical quenching is used to describe all quenching processes of PS II chlorophyll fluorescence that are not related to photochemistry. It mainly involves nonradiative, dissipative processes that are, for instance, induced by the build-up of a $\mathrm{pH}$ gradient across the thylakoid membrane (' $\Delta \mathrm{pH}$ quenching'). Non-photochemical quenching can only be determined when $F_{\mathrm{m}}$ is available from dark-adapted samples; it is defined as the normalised decrease of the maximal fluorescence yield with respect to the dark-adapted situation:

$$
q_{\mathrm{N}}=1-\frac{F_{\mathrm{m}}{ }^{\prime}-F_{0}{ }^{\prime}}{F_{\mathrm{m}}-F_{0}}
$$

(3) The photochemical yield of open PS II reaction centers, $F_{\mathrm{v}} / F_{\mathrm{m}}$. This ratio equals the product of the probabilities of excitation transfer between the antennae and PS II reaction center, and vice versa (e.g. Butler 1978). If non-radiative transfer in the reaction center is significantly smaller than the back transfer to the antennae pigments, the yield of photochemistry of the open PS II reaction centers is given by:

$$
\phi_{\mathrm{po}}=\frac{F_{\mathrm{v}}}{F_{\mathrm{m}}}=\frac{F_{\mathrm{m}}-F_{0}}{F_{\mathrm{m}}}
$$

It appears that $\phi_{\mathrm{po}}$ is a good indicator of photoinhibition. Light-induced damage to the PS II reaction center leads to lowering of the photochemical yield of the 
reaction centers (Bjorkman 1987). To assess photoinhibition, prolonged dark adaptation of the sample must be applied prior to the measurement to fully remove the effects of non-photochemical quenching. The recovery of the photosynthetic system from photoinhibitory effects often takes a long time, typically many hours.

(4) The photochemical efficiency of PS II per absorbed photon, or photon yield, $\phi_{p}$. The calculation is based on the assumption that the photon yield is given by the product of the effiency of an open PS II reaction center and the fraction of open reaction centers (Genty et al. 1989)

$$
\phi_{\mathrm{p}}=\frac{F_{\mathrm{m}}{ }^{\prime}-F_{0}{ }^{\prime}}{F_{m}{ }^{\prime}} \cdot \frac{F_{\mathrm{m}}{ }^{\prime}-F}{F_{m}{ }^{\prime}-F_{0}{ }^{\prime}}=\frac{F_{\mathrm{m}}{ }^{\prime}-F}{F_{\mathrm{m}}{ }^{\prime}}
$$

Since in steady state the flux of the electrons in PS I and PS II should be equal, the photon yield of PS II gives a good estimation of the efficiency of the linear electron flow in the photosynthetic apparatus. The overall rate of electron flow, $J_{\mathrm{e}}$ [in $\mu \mathrm{mol}$ e $(\mu \mathrm{mol} \text { PS II })^{-1}$ $\mathrm{s}^{-1}$, can be calculated by multiplying the photon yield with the amount of photons absorbed by PS II:

$$
J_{\mathrm{e}}=\phi_{\mathrm{p}} \cdot \sigma_{\mathrm{a}} \cdot I
$$

where $\sigma_{a}=$ the absorption cross section of PS $I_{i} I=$ the photon flux of the photosynthetically active radiation. As we were not able to determine $\sigma_{\mathrm{a}}$ directly, the (relative) $J_{\mathrm{e}}$ was estimated by the product $\phi_{\mathrm{p}} \cdot I$. This is a reasonable estimate: according to Kolber et al. (1988) the absorption cross section of Dunaliella tertiolecta does not change by more than 14 to $17 \%$ in the light range of 20 to $200 \mu \mathrm{E} \mathrm{m}^{-2} \mathrm{~s}^{-1}$.

The saturating pulse fluorescent approach can be applied in conjunction with the ${ }^{14} \mathrm{C}$ method, which is conventionally used for primary production measurements in the marine environment. An important advantage of the fluorescence technique over the ${ }^{14} \mathrm{C}$ method is that the former gives information on the instantaneous photosynthetic status and can be applied in situ (i.e. in the seawater at a particular location, measurements, for instance time series, can be performed). The measurement can be done directly as it does not require dark adaptation. To obtain absolute rates, calibration with ${ }^{14} \mathrm{C}$-incorporation measurements may be required.

An alternative optical approach, dubbed pump-andprobe fluorescence, can be used to measure properties similar to those measured with the saturating pulse fluorescence method used in this study (Falkowski \& Kiefer 1985, Falkowski \& Kolber 1990). The main difference between the 2 methods is the procedure used to determine $F_{\mathrm{m}}$ and $F_{\mathrm{m}}$ ', which can produce different results. In particular, as the pump-and-probe tech- nique only uses a single, short pump pulse, only a single turn-over of the PS II reaction centers is effected, which results in reduction of $Q_{A}$ only. In the saturating pulse approach a longer intense pulse is applied, which results in total reduction of $Q_{A}$ and of the $P Q$ pool. Therefore $F_{\mathrm{m}}$ and $F_{\mathrm{m}}$ ' in general will be somewhat lower in the pump-and-probe measurement. Thus, one expects to find a somewhat higher $q_{\mathrm{N}}$ and a somewhat lower $q_{p}$ for the pump-and-probe technique as compared to the saturating pulse approach. The trends determined by both methods, however, may be similar. A comparative study of both techniques, applied to phytoplankton photosynthesis, has been planned.

\section{EXPERIMENTAL APPLICATION}

The chlorophyte Dunaliella tertiolecta was grown at $15^{\circ} \mathrm{C}$ in $\mathrm{f} / 2$ medium (McLachlan 1975) under a $14 \mathrm{~h}$ light $10 \mathrm{~h}$ dark cycle. The cultures were kept in culture bottles, which were placed in plastic containers with white, diffuse reflecting walls, covered by suitable neutral density (gray) attenuation filters to obtain 7 different light intensities. An incubator (Gallenkamp Orbital INR-401) equipped with fluorescent tubes operating at a full light intensity of 300 to $400 \mu \mathrm{E} \mathrm{m} \mathrm{m}^{-2}$ $\mathrm{s}^{-1}$ was used. Light intensities in the containers were measured with a Photodyne 88 XLA spherical sensor. The intensities under which the cultures were grown are given in Table 1 .

The growth rate of the cultures was determined by means of a flow cytometer especially constructed for use with phytoplankton; the cytometer measured the concentration of particles with red chlorophyll fluorescence $(>670 \mathrm{~nm}$ ) under excitation with an Ar-ion laser operated at $529 \mathrm{~nm}$ (Hofstraat et al. 1990).

Saturating pulse fluorescence measurements were done with a pulse amplitude modulation fluorimeter (PAM, produced by Walz, Effeltrich, Germany, as developed by U. Schreiber; see Schreiber 1986). The PAM consists of 4 units, the settings of which were optimized before the actual experiment was started.

Table 1 Light intensities of photosynthetically active radiation under which the Dunaliella tertiolecta cultures were grown

\begin{tabular}{|cc|}
\hline Culture no. & Light intensity $\left(\mu \mathrm{E} \mathrm{m}^{-2} \mathrm{~s}^{-1}\right)$ \\
\hline 1 & 22.6 \\
2 & 42.1 \\
3 & 66.0 \\
4 & 92.3 \\
5 & 107.4 \\
6 & 137.3 \\
7 & 214.3 \\
\hline
\end{tabular}


Firstly, the modulated measuring light intensity provided by a pulsed LED in the 101-ED unit of the PAM, peaking at 650 to $660 \mathrm{~nm}$, had to be chosen so as to obtain sufficient sensitivity, yet be low enough to prevent induction of significant variable fluorescence Secondly, the actinic illumination from the KL-1500 lamp, controlled by the PAM 102 unit, was chosen to match the total light intensity under which the different cultures had been grown, as determined by a UDT PIN-10 photodiode. The matching was accomplished by applying the available settings of the actinic light source in combination with a number of Oriel neutral density filters. The spectral intensity distribution of the KL-1500 light source and that of the incubator were measured with a calibrated, spherical sensor, using $10 \mathrm{~nm}$ full-width-at-half-maximum bandpass filters to measure the absolute light intensity every $20 \mathrm{~nm}$. The intensity distributions of the 2 light sources did not match completely, the KL-1500 source being more intense in the orange and red part of the spectrum Thirdly, the intense, saturating light-pulse of the KL1500 light source, controlled by the PAM 103 unit, was optimized to ensure complete closure of the PS II reaction centers by observing the time resolved fluorescence signal as obtained by the modulated measuring light. At the same time actinic effects resulting from the saturating pulse should be absent; this was accomplished by application of a $700 \mathrm{~ms}$ pulse at an intensity of more than $2000 \mathrm{~W} \mathrm{~m}^{-2}$. Finally, far-red illumination (>730 nm) was applied to reopen the PS II reaction centers following a saturating pulse. Data acquisition and handling were performed by the dedicated program 'FLUORESC', developed at the Department of Plant Physiology, Wageningen Agricultural University.

The measuring procedure was carried out on a daily basis and commenced exactly $3 \mathrm{~h}$ after the light in the incubator was switched on. Samples were taken from the flasks in the incubator and put in an MKS-101 cuvette (provided by Walz) with minimal light exposure. Subsequently, measurement was started, with the actinic light intensity of the PAM matching that to which the sample had been exposed in the incubator. Due to the transfer to the measuring cuvette, the samples had been subjected to a change in light climate. Hence, each sample was irradiated with the actinic light source for $5 \mathrm{~min}$ before the actual measurement of $F_{\mathrm{m}}{ }^{\prime}$ and $F$. To confirm that after this adaptation time the steady-state condition had been reached, fluorescence and, at regular intervals, saturating pulse measurements were made during actinic illumination. Following this sequence the actinic light was switched off and another series of fluorescence and saturating pulse fluorescence readings was done in the dark, to check for possible measurement-induced quenching effects. For measurements in the dark, the pulse rate of the
LED in the 101-ED unit of the PAM, used for induction of fluorescence, was changed from $100 \mathrm{kHz}$ to $1.6 \mathrm{kHz}$ to prevent any actinic effects.

In a separate experiment, samples were darkadapted for $15 \mathrm{~min}$ to remove any energy-dependent quenching, and subsequently $F_{\mathrm{v}} / F_{\mathrm{m}}$ was measured to determine photoinhibition effects. $F_{\mathrm{v}} / F_{\mathrm{m}}$ was independent of the chlorophyll concentration between 0.5 and $40 \mu \mathrm{g}$ chlorophyll $\mathrm{ml}^{-1}$ (unpubl. results). Dilute samples (less than $10^{6}$ cells $\mathrm{ml}^{-1}$, corresponding to less than $1 \mu \mathrm{g}$ chlorophyll $\mathrm{ml}^{-1}$ ) were concentrated by centrifugation at $3000 \times g$ for $5 \mathrm{~min}$. Centrifugation did not affect the rate of photosynthesis.

\section{RESULTS AND DISCUSSION}

\section{Measurement of $\phi_{p}$ and $q_{p}$}

First, the applicability of the saturation pulse method for the measurement of $\phi_{\mathrm{p}}$ and $q_{\mathrm{P}}$ was examined; this measurement does not require dark adaptation of the phytoplankton. As can be inferred from Eq. (1), determination of $F_{\mathrm{m}}{ }^{\prime}, F$ and $F_{0}{ }^{\prime}$, all parameters which can be obtained under steady-state conditions, suffices for determination of $\phi_{\mathrm{p}}$ and $q_{\mathrm{p}}$.

The fluorescence induction kinetics obtained from 2 cultures that had been grown for $17 \mathrm{~d}$ under low and high light conditions, respectively, are shown in Fig. 2. Fig. 2a shows the fluorescence transient for low-lightadapted phytoplankton (Culture 3 ). At this light intensity the sample is still growing exponentially (see Fig. 3). The fluorescence induction kinetics indicate that the phytoplankton returns to a steady state almost immediately after the light pulse. In contrast, for the high-light-adapted cultures in the stationary phase (Culture 7, Fig. 3), a strong reduction of the effect of closing the reaction centers with a saturating pulse was observed once the sample was re-illuminated. The relatively short time during which the phytoplankton was kept in the dark prior to the measurement for the high-light case appears to have been sufficient to allow some recuperation of light-induced non-photochemical quenching. Also, for the high-light sample the saturating pulse appears to have caused some transient quenching effects.

The difference between the samples grown under relatively low and relatively high light regimes is striking (Table 2). The low-light sample, which is still in the exponential growth phase, shows a significantly higher photochemical efficiency than the high-light sample, which is in a steady state. Photochemical quenching, which can also be calculated from the direct measurement (under actinic illumination), appears to be somewhat higher for the low-light-adapted 

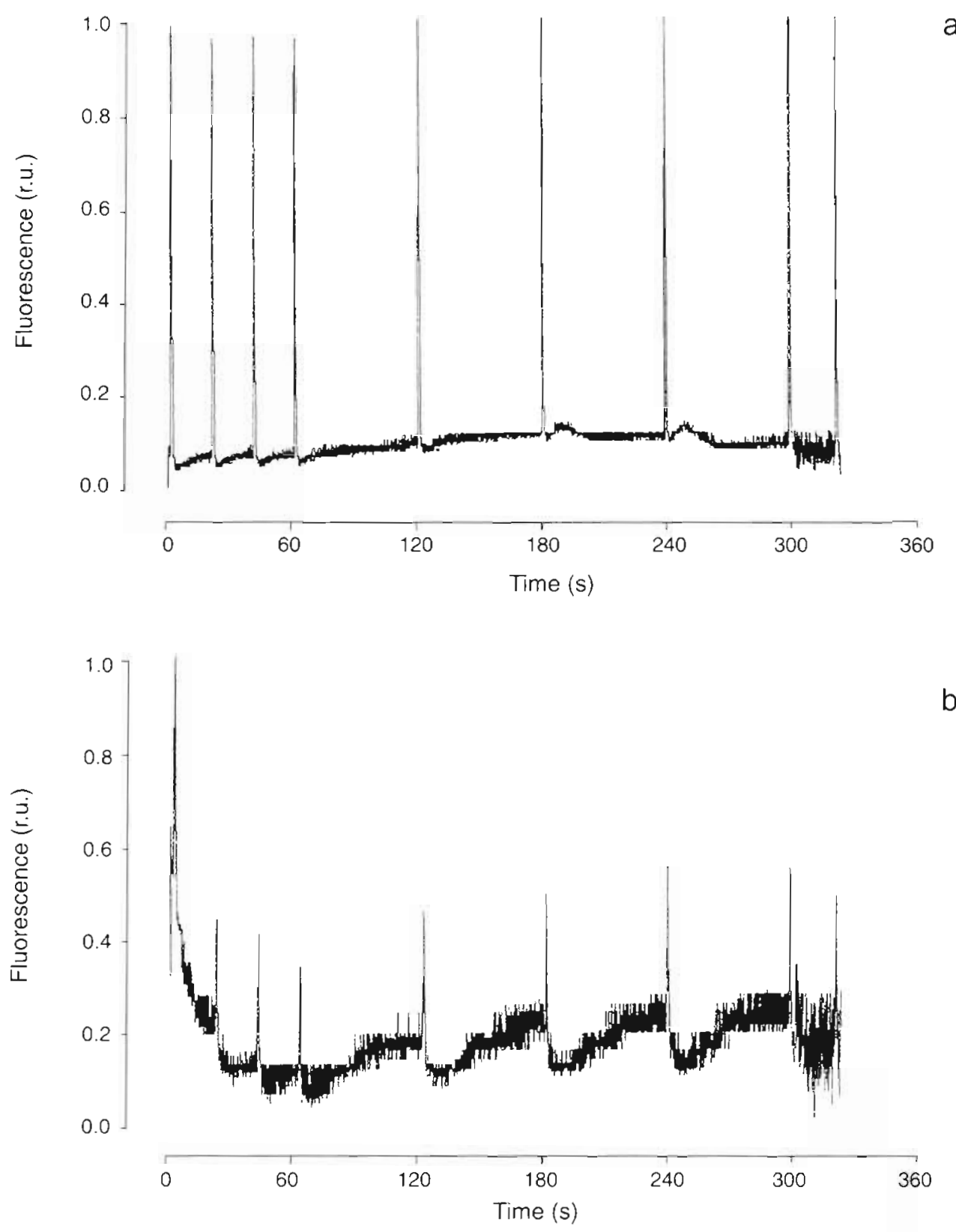

b

sample. Both observations indicate that photosynthetic energy conversion is more efficient in the low-light situation (see also Kolber et al. 1988). The curve in Fig $2 \mathrm{~b}$ also suggests a much higher contribution of non-photochemical quenching in the high-light-inten-

Table 2. Photosynthetic characteristics for the 2 Dunaliella tertiolecta cultures shown in Fig. 2

\begin{tabular}{|c|c|c|c|c|c|}
\hline $\begin{array}{c}\text { Culture } \\
\text { no. }\end{array}$ & $\begin{array}{l}\text { Light intensity } \\
\left(\mu \mathrm{E} \mathrm{m} \mathrm{m}^{-2} \mathrm{~s}^{-1}\right)\end{array}$ & $q_{N}$ & $q_{p}$ & $\phi_{p}$ & $F_{\mathrm{v}} / F_{\mathrm{t}}$ \\
\hline 3 & 66.0 & 0.37 & 0.96 & 0.58 & 0.69 \\
\hline 7 & 21.4 .3 & 0.61 & 0.81 & 0.25 & 0.42 \\
\hline
\end{tabular}

sity case. However, as no lengthy dark adaptation was done, data for $q_{\mathbb{N}}$ cannot be considered conclusive. Residual non-photochemical effects can still be present; dark adaptation will remove these effects, while more persistent photoinhibitory effects will remain. Non-photochemical (or energy-dependent) quenching will be more important for the high-light case, so that the difference in the values obtained for $q_{N}$ given in Table 2 will be more pronounced when the samples are dark adapted for a longer time.

When cultures grown under different light intensities are followed over time, observations similar to those mentioned above are made. In Fig. 4 a the development of $\phi_{p}$ with time is presented. Five days after 
incubation was started, the 6 cultures (Cultures 2 to 7 ; Table 1) had more or less similar values for $\phi_{\mathrm{p}}(0.64$ to 0.74$)$. All cultures were growing exponentially. After $17 \mathrm{~d}$ a clear difference, depending on the light intensity, had become apparent. At that time $\phi_{\mathrm{p}}$ ranged from 0.30 to 0.67 . The 2 cultures grown under the lowest light intensities were still in exponential growth after $17 \mathrm{~d}$. Indeed, the value of $\phi_{p}$ determined for these cultures appeared to have hardly decreased. Cultures 4 and 5 showed exponential growth until Day 13. Cultures 6 and 7 entered the stationary (growth) phase around Day 11, the time at which $\phi_{p}$ started to decrease.

$\phi_{p}$ only gives an estimate of the efficiency of the electron flow in PS II. The overall rate of electron flow, $J_{\mathrm{e}}$, which represents the photosynthetic activity of the phytoplankton, can be calculated by multiplying $\phi_{p}$ by the amount of photons absorbed by PS II. If we assume equal absorption cross sections for the different cultures, the (relative) $J_{e}$ can be approximated by the product of $\phi_{p}$ and the integrated intensity of the light used to culture the samples. Fig. $4 \mathrm{~b}$ shows the product $\phi_{\mathrm{p}} \cdot I$ as a function of incubation time for the different cultures. $\phi_{p} \cdot I$ is an approximation of the linear electron flow in PS II, assuming that the absorption cross section is equal for all cultures. Clearly, the highlight cultures show significantly higher overall rates in the first phase of the experiment. The 2 cultures grown at the lowest light intensities show constant $J_{\mathrm{e}}$ during the course of the experiment.

A relation between the photosynthetic activity and the growth rate of the phytoplankton cultures is expected. Fig. 5 shows the correspondence between $\phi_{\mathrm{p}} \cdot I$ (the estimated electron transport) and the measured growth rate of the cultures during the exponential growth phase. The relation is clear: at low electron transport rates (i.e. at light levels below $100 \mu \mathrm{E} \mathrm{m}^{-2} \mathrm{~s}^{-1}$ ) the exponential growth rate is more or less determined by the light intensity. At higher light levels the growth rate approaches a limiting exponential value of about 1 . Extrapolation to zero electron transport $\left(\phi_{p} \cdot I=0\right)$ indicates no growth, as expected.
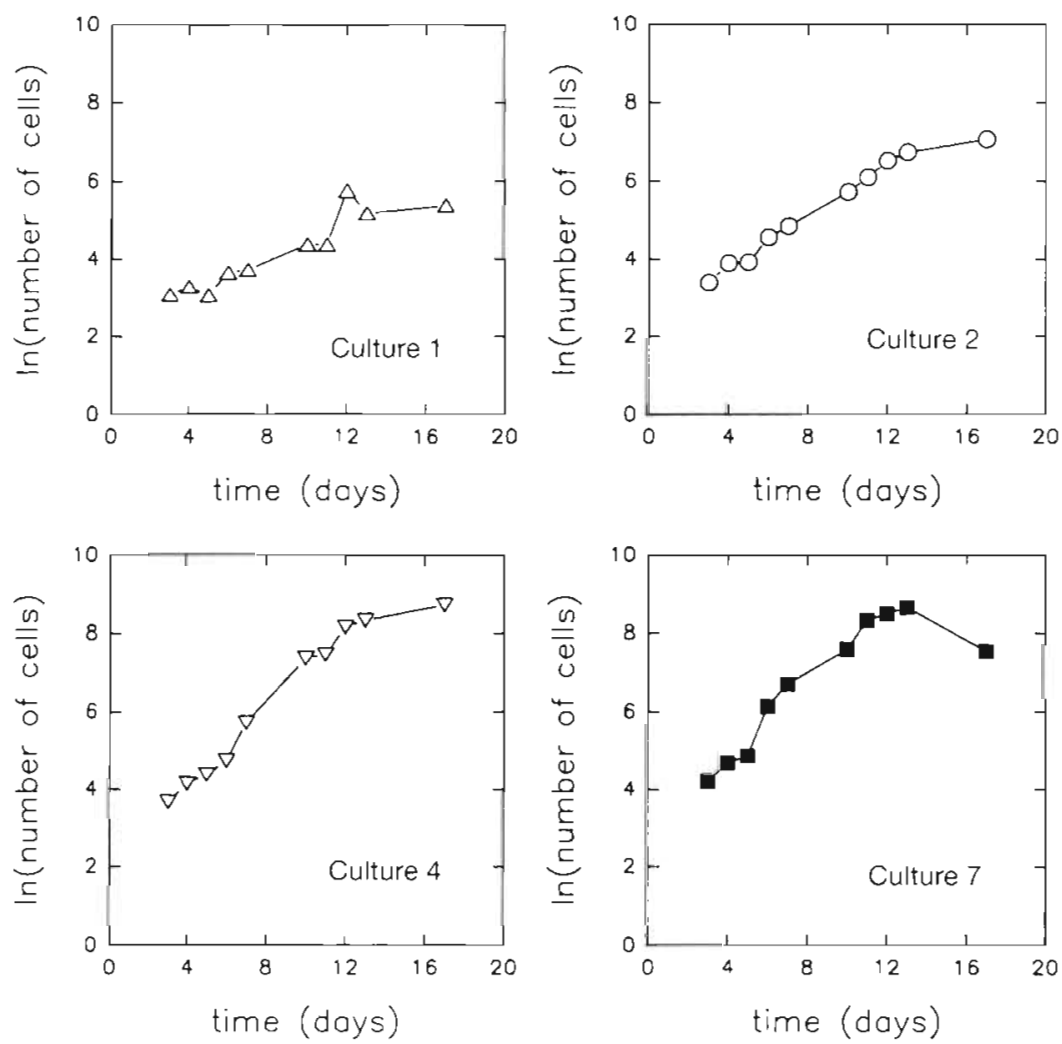

Fig. 3. Increase in Dunaliella tertiolecta cell numbers in the cultures, as determined by flow cytometry. Curves are shown for 4 typical cultures; the corresponding light intensities are given in Table 1
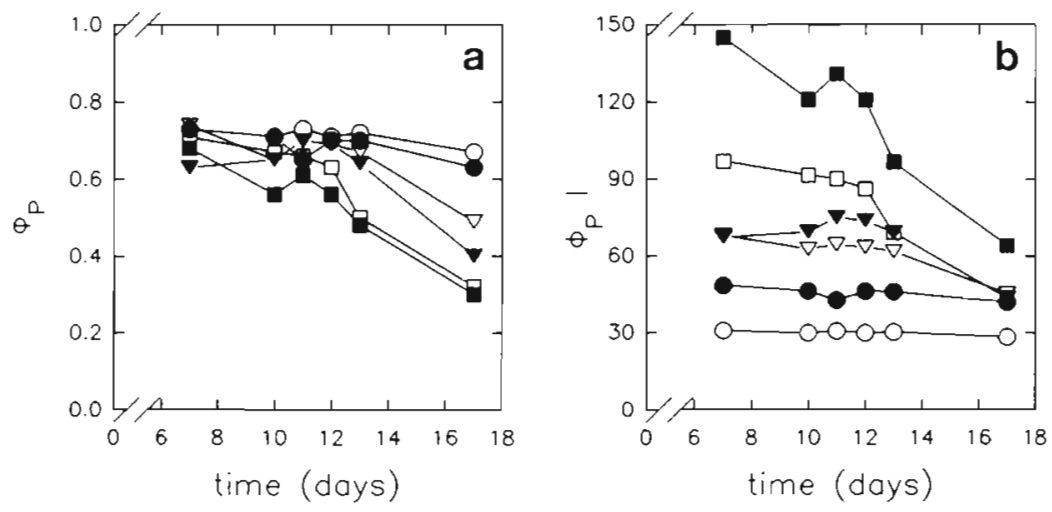

Fig. 4. Development of (a) $\phi_{\mathrm{p}}$ and (b) $\phi_{\mathrm{p}} \cdot I$ with time for Dunaliella tertiolecta Cultures 2 to 7 , grown under the light intensities given in Table 1. (O) Culture

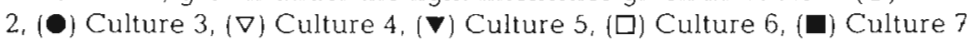




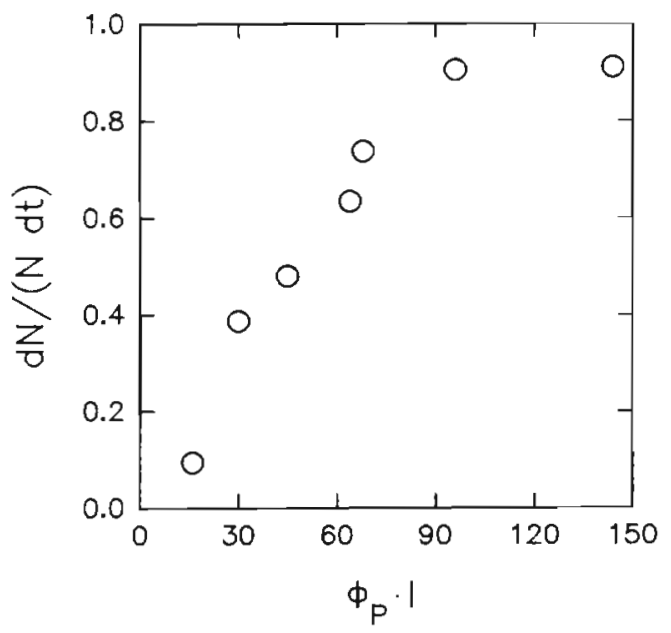

Fig. 5. Relation between $\phi_{p} \cdot I$ and Dunaliella tertiolecta growth rate. Both $\phi_{p}$ and growth rate were determined as averages during the exponential growth phase $\mathrm{N}=$ cell number

(Peterson 1980, Dring \& Jewson 1982). Indeed, Genty et al. (1989) have reported very good correlations between the photochemical yield as determined by the saturating pulse technique and the quantum yield of $\mathrm{CO}_{2}$ assimilation as determined by the ${ }^{14} \mathrm{C}$-incorporation technique. The saturating pulse technique and the pump-probe technique (Falkowski \& Kolber 1990) have several advantages over the latter method. Firstly, they do not require radioactive substances. Secondly, they can be applied directly, without incubation as required for the ${ }^{14} \mathrm{C}$ technique. Therefore, they are less cumbersome and more efficient and can even be applied in situ, so that the photosynthetic activity of phytoplankton can be monitored, for example, over a certain period of time. On the other hand, the ${ }^{14} \mathrm{C}$ method directly monitors the incorporation of $\mathrm{CO}_{2}$ into the phytoplankton and therefore provides more straightforward information on the photosynthetic energy conversion process. The fluorescence techniques described here give direct information on photosynthetic electron flow, as they are based solely on measurement of PS II fluorescence. With these techniques, therefore, it is not possible to distinguish between energy storage in the Calvin cycle and that in other assimilation pathways (e.g. N reduction, photorespiration). Another advantage of the ${ }^{14} \mathrm{C}$ method is that it provides a time-averaged (e.g. daily) rate of primary production, which can be useful for certain applications. In this respect the 2 approaches, the fluorescence-based methods and the ${ }^{14} \mathrm{C}$ method, are complementary. Presumably, in future applications one would perform most measurements with the fluorescence technique, but would take several samples for ${ }^{14} \mathrm{C}$-incorporation analysis to calibrate $\phi_{\mathrm{p}}$ in terms of biomass production.

\section{Determination of photoinhibition}

As indicated above, photoinhibition can be estimated from the ratio of the variable and maximum fluorescence $F_{\mathrm{v}} / F_{\mathrm{m}}=\left(F_{\mathrm{mi}}-F_{0}\right) / F_{\mathrm{m}}$. This ratio is a measure of the photochemical yield in open reaction centers (Björkman 1987). Due to primary damage to the PS II reaction center, the variable fluorescence especially is reduced. Photoinhibition can be determined only when all transient quenching processes have been allowed to relax. Hence, following transfer of the samples from the illuminated containers, the samples are dark adapted for $15 \mathrm{~min}$, which is sufficient to remove any photochemical or energy-dependent quenching. Then $F_{0}$ and $F_{\mathrm{m}}$ are measured, the latter by using a short pulse of saturating light.

In Fig. $6 F_{\mathrm{v}} / F_{\mathrm{m}}$ is shown for Cultures 2 to 7 (Table 1 ) during the course of the experiment. In the first phase of the experiment, when all the cultures are still growing exponentially, differences in the ratio are small: all cultures show values between 0.73 and 0.80 . However, after $12 \mathrm{~d}$ of growth, significant differences are observed. In particular the cultures grown under highlight conditions start to give indications of photoinhibitory effects, the $F_{\mathrm{v}} / F_{\mathrm{m}}$ ratio dropping to a mere 0.4 for the 2 cultures exposed to the highest light conditions. At the same time the growth curves for these cultures show clear non-exponential growth behaviour, and even a small decline in cell numbers for the last day of the experiment. The fact that in exponentially growing cultures $F_{\mathrm{v}} / F_{\mathrm{m}}$ is almost as high as 0.8 suggests that even at the relatively high cell concentrations used in the measurements, saturation of fluores-

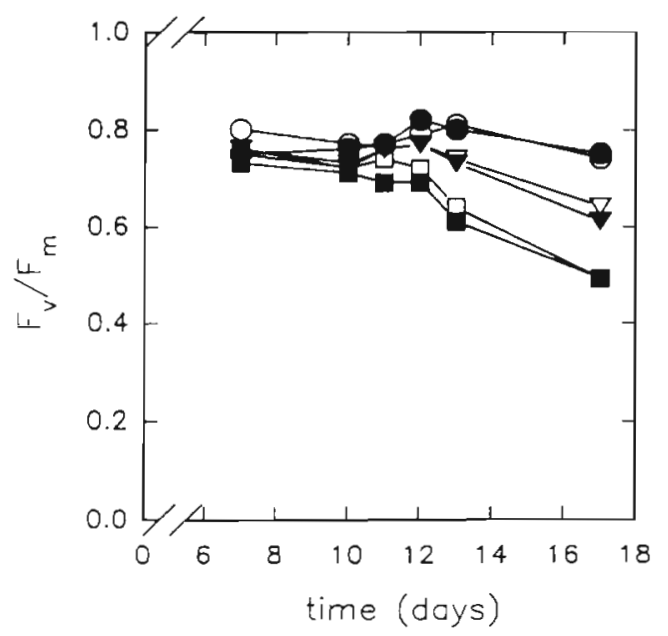

Fig. 6. Development of the parameter $F_{\mathrm{v}} / F_{\mathrm{m}}$, representative of the extent of photoinhibition, with tume for Dunaliella tertiolecta Cultures 2 to 7 according to Table 1. (O) Culture 2, (-) Culture $3,(\nabla)$ Culture $4,(\nabla)$ Culture $5,(\square)$ Culture 6, (a) Culture 7 
cence and reabsorption effects are not important. For other phytoplankton species such as Phaeodactylum tricornutum and Rhodomonas sp., $F_{\mathrm{v}} / F_{\mathrm{m}}$ values of 0.7 and higher have been observed (C. Geel unpubl.). $F_{\mathrm{v}} / F_{\mathrm{m}}$ is highly correlated with $\phi_{\mathrm{p}}$, which suggests that chlororespiration should have only a minor influence.

The example shown in Fig. 6 illustrates the potential of this technique as a simple means of monitoring photoinhibitory effects in phytoplankton. Using the saturating pulse fluorescence technique $P / I$ curves can easily be made. As the measurement is based on a relative approach, the method appears extremely robust. The repeatability of the determination of $F_{\mathrm{v}} / F_{\mathrm{m}}$ is better than $1 \%$. However, due to the insensitivity of the PAM fluorimeter applied to phytoplankton measurement, it is crucial to implement corrections for the effect of stray light. This effect cannot be neglected; it has a particularly strong influence on the determination of $F_{0}$, but it can easily be corrected for.

\section{Modifications for phytoplankton measurement}

A final problem of the saturating pulse technique as realized in the PAM fluorimeter is that the instrument was specifically designed for studies on higher plants. Therefore, it is not sufficiently sensitive for study of phytoplankton. In our study we found that the concentration of Dunaliella tertiolecta should exceed $10^{6}$ cells $\mathrm{ml}^{-1}$ in order for good results to be obtained with the PAM. At this concentration $\phi_{p}$ can still be determined with a repeatability of better than $5 \%$, as the determination is a relative one and therefore relatively robust. However, for applications on field samples or for dilute cultures the sensitivity is 3 to 4 orders of magnitude too low. In the laboratory the range of the instrument can be extended for most phytoplankton species by centrifugation. For field samples this approach cannot be applied. However, the sensitivity of the instrument can probably be improved significantly by relatively straightforward modifications. The present instrument was designed for application to plants and leaves. It uses excitation in the 650 to $660 \mathrm{~nm}$ region and detection at $>710 \mathrm{~nm}$. For phytoplankton, in which the chlorophyll concentration is much lower, excitation in the blue and detection at $>670 \mathrm{~nm}$ would already yield a tremendous increase in sensitivity. In addition, the volume of interaction between light and phytoplankton is very small. For phytoplankton dispersed in surface water at relatively low concentrations a much larger volume of interaction would also lead to a significant increase in sensitivity. Finally, some improvements in data acquisition and transfer could be implemented.

Recent experiments with a modified PAM instrument, incorporating the changes suggested above and, in addition, a pulsed Xenon flash lamp as excitation source have shown that the required improvements in sensitivity can indeed be realized (U. Schreiber pers. comm.). A modified PAM instrument is presently being built, and will be used for further study.

\section{CONCLUSIONS}

The saturating pulse fluorescence technique appears to be a method that can be successfully used for the non-destructive measurement of important photosynthetic characteristics of Dunaliella tertiolecta. It allows for the simple and direct measurement of photosynthetic efficiency, of photoinhibitory effects and of fluorescence quenching processes. For some parameters the samples must be dark adapted prior to the measurement. The saturating pulse fluorescence approach, and the pump-and-probe technique (Falkowski \& Kolber 1990), seem to offer useful extensions to the conventionally used techniques. For instance, ${ }^{14} \mathrm{C}$-uptake measurements require the use of radioactive material, supply time-integrated photosynthetic data and cannot be used for in situ monitoring applications. Oxygen measurements, which may be used for in situ applications, are not sufficiently sensitive for marine use.

However, several improvements still have to be made in the design of the PAM fluorimeter. First and foremost, the sensitivity of the instrument, which was developed for plant studies, must be improved to enable measurement of field samples. Also, measurements performed with the saturating pulse technique have to be compared with those from conventional techniques, as has already been done (with good results) for plants.

Acknowledgements. Messrs IJ. A. Althuis, M. L. Latuhihin, L. Peperzak, M. E. J. de Vreeze and W. J. M. van Zeijl are gratefully acknowledged for their contributions to the experiments described in this paper

\section{LITERATURE CITED}

Björkman, O. (1987). Low temperature chlorophyll fluorescence in leaves and its relation to photon yield of photosynthesis in photoinhibition. In: Kyle, D. J., Osmond, O. B., Arntzen, C. J. (eds.) Photoinhibition. Elsevier Science Publishers, Amsterdam, p. 123-44

Briantais, J.-M., Vernotte, C., Krause, G. H., Weis, E. (1986). Chlorophyll a fluorescence of higher plants: chloroplast and leaves. In: Govindjee, Amesz, J., Fork, D. C. (eds.) Light emission by plants and bacteria. Academic Press, New York, p. 539

Bryan, J. R., Riley, J. P., Williams, P. J. le B. (1976). A Winkler procedure for making precise measurements of oxygen concentration for productivity and related studies. J. exp. mar. Biol. Ecol. 21: 191-197 
Butler, W. L. (1978). Energy distribution in the photochemical apparatus of photosynthesis. A. Rev. Plant Physiol. 29: $345-378$

Butterwick, C., Heaney, S. I., Talling, J. F. (1982). A comparison of eight methods for estimating the biomass and growth of planktonic algae. Br. Phycol. 17:69-79

Dring, M. J., Jewson, D. H. (1982). What does ${ }^{14} \mathrm{C}$ uptake by phytoplankton really measure? A theoretical modelling approach. Proc. R. Soc. Lond. B 214: 351-368

Falkowski, P., Kiefer, D. A. (1985). Chlorophyll a fluorescence in phytoplankton: relationship to photosynthesis and biomass. J. Plankton Res. 7: 715-731

Falkowski, P. G., Kolber, Z. (1990). Phytoplankton photosynthesis in the Atlantic Ocean as measured from a submersible pump and probe fluorometer in situ. In: Baltscheffsky, M. (ed.) Current research in photosynthesis, Proceedings of the 8th International Conference on Photosynthesis, Vol. 4. Kluwer, Dordrecht, p. 923-926

Falkowski, P. G., Wyman, K., Ley, A. C., Mauzerall, D. C. (1986). Relationship of steady state photosynthesis to fluorescence in eucaryotic algae. Biochem. biophys. Acta 849 . $183-192$

Genty, B., Briantais, J.-M., Baker, N. R. (1989). The relationship between the quantum yield of photosynthetic electron transport and quenching of chlorophyll fluorescence. Biochim. biophys. Acta 990: 87-92

Heaney, S. I. (1978). Some observations on the use of the in vivo fluorescence technique to determine chlorophyll-a in natural populations and cultures of freshwater phytoplankton. Freshwat. Biol. 8: 115-126

Hilton, J., Rigg, E., Jaworski, G. (1989). Algal identification using in vivo fluorescence spectra. J. Plankton Res. 11: $65-74$

Hofstraat, J. W. van Zeijl, W. J. M., Peeters, J. C. H., Peperzak, L., Dubelaar, G. B. J. (1990). Flow cytometry and other optical techniques for characterization and quantification of phytoplankton in seawater. In: Nielsen, H. O. (ed.) Environment and pollution measurement sensors and systems. SPIE Proc. 1269: 116-133

Kolber, Z., Zehr, J., Falkowski, P. (1988). Effects of growth irradiance and nitrogen limitation on photosynthetic energy conversion in photosystem II. Plant Physiol. 88: $923-929$
Lichtenthaler, H. K., Rinderle, U. (1988). The role of chlorophyll fluorescence in the detection of stress conditions in plants. CRC Cnt. Rev. Chem. 19: S29-S85

McLachlan, J. (1975). Growth media marine. In: Stein, J. (ed.) Handbook of phycological methods. Cambridge University Press, Cambridge, p. 25-51

Peterson, B. J. (1980). Aquatic primary productivity and the ${ }^{14} \mathrm{C}-\mathrm{CO}_{2}$ method: a history of the productivity problem. A. Rev. Ecol. Syst. 11.359-385

Renger, G., Schreiber, U. (1986). Practical applications of fluorometric methods to algae and higher plant research. In: Govindjee, Amesz, J., Fork, D. C. (eds.) Light emission by plants and bacteria. Academic Press, New York, p. 587

Schreiber, U. (1983). Chlorophyll fluorescence yield changes as a tool in plant physiology. I. The measuring system. Photosynth. Res. 4: 361-373

Schreiber, U. (1986). Detection of rapid induction kinetics with a new type of high-frequency modulated chlorophyll fluorometer. Photosynth. Res. 9: 261-271

Schreiber, U., Schliwa, U., Bilger, W. (1986). Continuous recording of photochemical and non-photochemical fluorescence quenching with a new type of modulation fluoro. meter. Photosynth. Res. 10: 51-62

Strasser, R. J., Butler, W. L. (1977). The yield of energy transfer and the spectral distribution of excitation energy in the photochemical apparatus of flashed bean leaves. Biochim. biophys. Acta 462: 295

Van Kooten, O., Snel, J. F. H. (1990). The use of chlorophyll fluorescence nomenclature in plant stress physiology. Photosynth. Res. 25: 147-150

Yentsch, C. S., Phinney, D. A. (1985a). Fluorescence spectral signatures for studies of marine phytoplankton. In: Zirino, A. (ed.) Mapping strategies in chemical oceanography. Advances in Chemistry Series, No. 209. American Chemical Society, Washington, DC, p. 259

Yentsch, C. S., Phinney, D. A. (1985b). Spectral fluorescence: an ataxonomic tool for studying the structure of phytoplankton populations. J. Plankton Res. 7: 617-632

Yentsch, C. S., Yentsch, C. M. (1979). Fluorescence spectral signatures: the characterization of phytoplankton populations by the use of excitation and emission spectra. J. mar. Res. 37: 471-483

Manuscript first received: March 23, 1993

Revised version accepted: August 24, 1993 